\title{
Communication
}

\section{Synthesis and Antifungal Activity of Carabrone Derivatives}

\author{
Jun-Tao Feng ${ }^{1, *}$, Zhi-Qing Ma ${ }^{1}$, Jiang-Hua $\mathrm{Li}^{1}$, Jun He ${ }^{1}$, Hui Xu ${ }^{1,2, *}$ and Xing Zhang ${ }^{1}$
}

1 Research and Development Center of Biorational Pesticide, College of Plant Protection, Northwest A\&F University, Yangling 712100, China

2 Laboratory of Pharmaceutical Design \& Synthesis, College of Sciences, Northwest A\&F University, Yangling 712100, China

* Author to whom correspondence should be addressed; E-Mails: fengjt67@hotmail.com (J.-T.F.); orgxuhui@nwsuaf.edu.cn (H.X.)

Received: 6 July 2010; in revised form: 11 August 2010 / Accepted: 23 August 2010 /

Published: 16 September 2010

\begin{abstract}
Nine derivatives 6-14 of carabrone (1) were synthesized and tested in vitro against Colletotrichum lagenarium Ell et Halst using the spore germination method. Among all of the derivatives, compounds 6-8 and 12 showed more potent antifungal activity than 1. Structure-activity relationships (SAR) demonstrated that the $\gamma$-lactone was necessary for the antifungal activity of $\mathbf{1}$, and the substituents on the C-4 position of $\mathbf{1}$ could significantly affect the antifungal activity.
\end{abstract}

Keywords: carabrone; structural modification; synthesis; antifungal activity

\section{Introduction}

Carabrone (1, Figure 1), containing cyclopropane and sesquiterpene lactone moieties, was first isolated from the fruits of Carpesium abrotanoides [1], and is widely distributed in feverfew and other plant species [2-9]. It was demonstrated that compound 1 displays cytotoxic [10], antibacterial [11,12], and antitumor activity [13]. In our course of screening for novel naturally occurring phytofungicides from the plants in northwestern China, compound $\mathbf{1}$ was obtained from Carpesium macrocephalum, and exhibited antifungal activities in vitro and in vivo against Botrytis cinerea, Colletotrichum lagenarium, and Erysiphe graminis [14]. Subsequently, we prepared four derivatives (2-5, Figure 1) from 1, and found that the 11,13-double bond and the carbonyl group on the C-4 position of 1 are two 
active sites $[15,16]$. In order to further investigate the effect of lactone and substituents on the C-4 position of $\mathbf{1}$ on the antifungal activity, herein we synthesized nine new carabrone derivatives 6-14 as potential antifungal agents.

Figure 1. The chemical structures of carabrone and its derivatives.
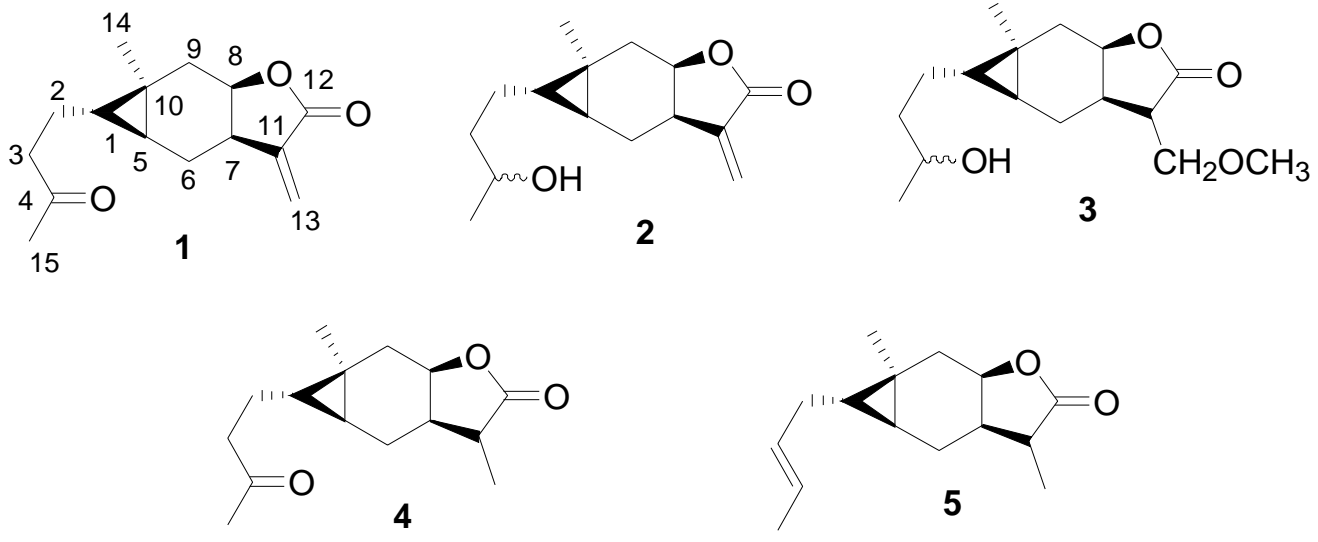

\section{Results and Discussion}

Nine carabrone derivatives 6-14 were synthesized as shown in Scheme 1. Compound 6 was prepared by the reaction of 2,4-dinitrophenyl hydrazine (DNPH) and $\mathbf{1}$ in the presence of hydrogen chloride $(\mathrm{HCl})$. Benzhydrazide or semicarbazide reacted with $\mathbf{1}$ to give compounds $\mathbf{7}$ and $\mathbf{8}$, respectively. Compound $\mathbf{9}$ was prepared from 1 with dry $\mathrm{HCl}$. Compound $\mathbf{1 0}$ was synthesized by the reduction of the carbonyl group of 1 in the presence of $\mathrm{NaBH}_{4}$, followed by chlorination of the 4-OH group of $\mathbf{2}$ with thionyl chloride $\left(\mathrm{SOCl}_{2}\right)$. Compound $\mathbf{2}$ reacted with acyl chlorides in the presence of pyridine to afford compounds 11-14. All compounds were characterized by ${ }^{1} \mathrm{H}-\mathrm{NMR}$, IR, and HR-MS spectra.

Scheme 1. The synthetic route to carabrone derivatives 6-14.

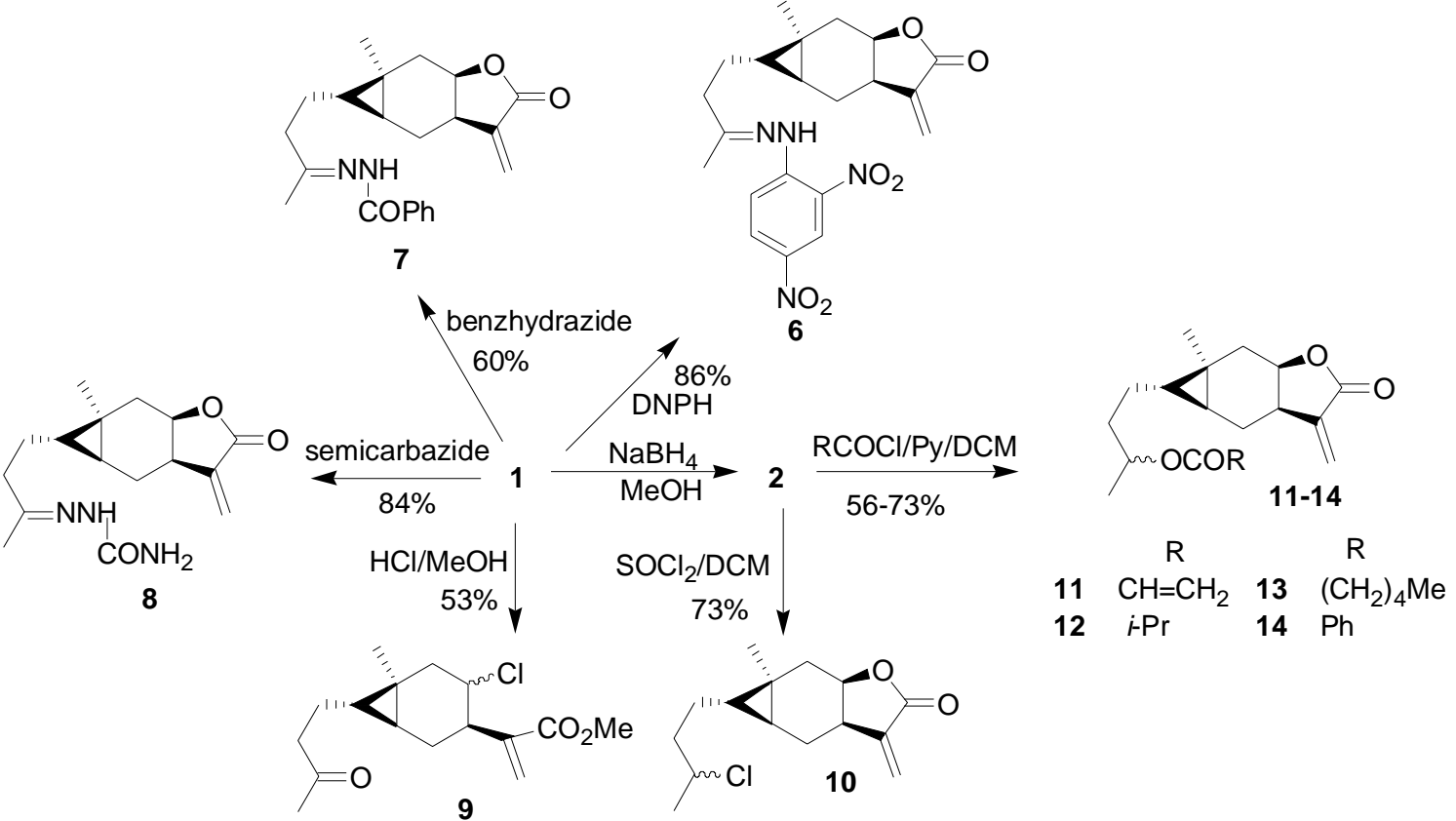


The antifungal activity was assayed in vitro against Colletotrichum lagenarium Ell et Halst by the spore germination method. Chlorothalonil was used as a positive control. As described in Table 1, compounds 6-8 exhibited the most potent antifungal activity with the $\mathrm{EC}_{50}$ values of 2.24, 4.32 and $3.03 \mu \mathrm{g} / \mathrm{mL}$, respectively, i.e., the antifungal activity of $\mathbf{6 , 7}$ and $\mathbf{8}$ was $1.5-3$ times more potent than that of $\mathbf{1}$. However, the antifungal activity of other compounds was 1-8 times less than that $\mathbf{1}$. Obviously, substituents on the C-4 position of 1 could significantly affect the antifungal activity. For example, introducing the hydrazone substituents on the C-4 position of $\mathbf{1}$ lead to the most potent compounds (e.g., 6-8), while when other substituents, such as the hydroxy group, chloro atom, and ester groups (except isobutyryloxy group), were introduced on the $\mathrm{C}-4$ position of $\mathbf{1}$, the corresponding compounds showed the less potent activity than 1 (e.g., 10, 11, 13 and 14). Interestingly, when the isobutyryloxy group was introduced on the C-4 position of $\mathbf{1}$ to give $\mathbf{1 2}$, the $\mathrm{EC}_{50}$ value of $\mathbf{1 2}$ was $6.39 \mu \mathrm{g} / \mathrm{mL}$, which was more potent than that of $\mathbf{1}$. Meanwhile, compound $\mathbf{1}$ was nearly eightfold more potent than $9\left(\mathrm{EC}_{50} 7.10 \mu \mathrm{g} / \mathrm{mL}\right.$ for 1 vs. $\mathrm{EC}_{50} 56.30 \mu \mathrm{g} / \mathrm{mL}$ for 9$)$. This demonstrated that the $\gamma$ lactone was necessary for the antifungal activity of $\mathbf{1}$, and opening the lactone would lead to a less potent compound (1 vs. 9).

Table 1. Inhibition rates of carabrone derivates (6-14) against spore germination of Colletotrichum lagenarium. ${ }^{a}$

\begin{tabular}{ccccc}
\hline Compd. & $\begin{array}{c}\text { Regression equation } \\
(\mathbf{Y}=\mathbf{a}+\mathbf{b X})\end{array}$ & $\mathbf{r}$ & $\mathbf{E C}_{\mathbf{5 0}} \boldsymbol{b}^{\boldsymbol{}}(\boldsymbol{\mu} \mathbf{g} / \mathbf{m L})$ & $\mathbf{E C}_{\mathbf{5 0} \mathbf{9 5} \% \mathbf{C L} /(\boldsymbol{\mu g} / \mathbf{m L})}$ \\
\hline $\mathbf{1}$ & $\mathrm{Y}=3.6090+1.6337 \mathrm{X}$ & 0.9974 & 7.10 & $6.19 \sim 8.02$ \\
$\mathbf{6}$ & $\mathrm{Y}=4.5130+1.3891 \mathrm{X}$ & 0.9923 & 2.24 & $1.97 \sim 2.55$ \\
$\mathbf{7}$ & $\mathrm{Y}=3.4038+2.5118 \mathrm{X}$ & 0.9817 & 4.32 & $3.81 \sim 4.85$ \\
$\mathbf{8}$ & $\mathrm{Y}=4.3577+1.3351 \mathrm{X}$ & 0.9979 & 3.03 & $2.58 \sim 3.55$ \\
$\mathbf{9}$ & $\mathrm{Y}=3.1867+1.0358 \mathrm{X}$ & 0.9942 & 56.30 & $42.95 \sim 73.80$ \\
$\mathbf{1 0}$ & $\mathrm{Y}=3.2442+1.1736 \mathrm{X}$ & 0.9969 & 31.34 & $26.08 \sim 37.66$ \\
$\mathbf{1 1}$ & $\mathrm{Y}=4.2780+0.6720 \mathrm{X}$ & 0.9882 & 10.78 & $9.16 \sim 12.68$ \\
$\mathbf{1 2}$ & $\mathrm{Y}=4.3568+0.7209 \mathrm{X}$ & 0.9920 & 6.39 & $5.33 \sim 7.65$ \\
$\mathbf{1 3}$ & $\mathrm{Y}=3.7775+0.9365 \mathrm{X}$ & 0.9970 & 20.20 & $16.85 \sim 24.22$ \\
$\mathbf{1 4}$ & $\mathrm{Y}=3.7676+1.0011 \mathrm{X}$ & 0.9962 & 17.02 & $14.41 \sim 20.11$ \\
chlorothalonil $^{c}$ & $\mathrm{Y}=5.1247+1.0081 \mathrm{X}$ & 0.9935 & 0.75 & $0.63 \sim 0.90$ \\
\hline
\end{tabular}

${ }^{a}$ Values are means of three separate experiments; ${ }^{b} \mathrm{EC}_{50}(50 \%$ effective concentration), concentration of compound that reduces spore germination by $50 \% ;{ }^{c}$ Chlorothalonil was used as a positive control.

\section{Experimental}

\subsection{General}

All the solvents were of analytical grade and the reagents were commercially available. Thin-layer chromatography (TLC) and silica gel-column chromatography were performed with silica gel plates using silica gel $60 \mathrm{GF}_{254}$, and 200-300 mesh (Qingdao Haiyang Chemical Co., Ltd., China). Melting points were determined on a digital melting-point apparatus and uncorrected. All compounds were characterized by proton nuclear magnetic resonance $\left({ }^{1} \mathrm{H}-\mathrm{NMR}\right)$, high-resolution mass spectra (HRMS), mass spectra (MS-ESI), and infrared spectra (IR), respectively. 


\subsection{Synthesis}

(3aR, 4aS, 5S, 5aR, 6aR)-5-(3-keto 2, 4-dinitrophenyl hydrazone-butyl)-5a-methyl-3-methylene-3a, 4, $4 a$, 5, 6, 6a-hexahydrocyclopropa[f]benzofuran-2-one (6). A mixture of compound 1 (124 mg, $0.5 \mathrm{mmol}), 2$,4-dinitrophenyl hydrazine (DNPH, $39.6 \mathrm{mg}, 2 \mathrm{mmol})$ and hydrochloric acid $(0.2 \mathrm{~mL}$, $6 \mathrm{~mol} / \mathrm{L})$ in anhydrous methanol $(\mathrm{MeOH}, 10 \mathrm{~mL})$ was reacted at $60^{\circ} \mathrm{C}$ until a precipitate formed. The reaction mixture was then filtered, and the filtrate was evaporated under educed pressure. The residue was recrystallized in dimethyl sulfoxide (DMSO) to produce 6 as a yellow solid. Yield: 86\%, m.p. 181-182 ${ }^{\circ} \mathrm{C}$ [17]; IR (KBr) cm ${ }^{-1}: 3321,2956,1756,1593,1530,1345 ;{ }^{1} \mathrm{H}-\mathrm{NMR}\left(400 \mathrm{MHz}, \mathrm{CDCl}_{3}\right)$ $\delta: 10.79(\mathrm{~s}, 1 \mathrm{H},=\mathrm{NNH}), 8.84\left(\mathrm{~m}, 1 \mathrm{H}, \mathrm{H}-3^{\prime}\right), 8.35\left(\mathrm{~d}, J=6.8 \mathrm{~Hz}, 1 \mathrm{H}, \mathrm{H}-5^{\prime}\right), 7.82(\mathrm{~d}, J=10.0 \mathrm{~Hz}, 1 \mathrm{H}$, H-6'), 5.99 (d, $J=2.4$ Hz, 1H, H-13), 5.62 (d, $J=2.4$ Hz, 1H, H-13), 4.80 (m, 1H, H-8), 3.31 (m, 2H), 3.18 (m, 1H, H-7), 2.43 2.55 (m, 1H), 2.26 2.35 (m, 1H), 2.05 (s, 3H, H-15), 1.54 1.64 (m, 2H), 1.05 (s, 3H, H-14), 0.85 0.91 (m, 2H), 0.53 (m, 1H, H-5), 0.35 (m, 1H, H-1); HR-MS (ESI): $m / z$ calcd for $\mathrm{C}_{21} \mathrm{H}_{25} \mathrm{~N}_{4} \mathrm{O}_{6}\left([\mathrm{M}+\mathrm{H}]^{+}\right), 429.1769$; found, 429.1763 .

(3aR, 4aS, 5S, 5aR, 6aR)-5-(3-keto benzoyl hydrazone-butyl)-5a-methyl-3-methylene-3a, 4, 4a, 5, 6, 6a-hexahydrocyclopropa[f] benzofuran-2-one (7). A mixture of 1 (125 mg, $0.5 \mathrm{mmol}$ ), benzhydrazide ( $82 \mathrm{mg}, 0.6 \mathrm{mmol})$, and 1-2 drops of HOAc in absolute ethanol $(10 \mathrm{~mL})$ was stirred at $80^{\circ} \mathrm{C}$. After $3 \mathrm{~h}$, the solvent was removed under reduced pressure to give a residue, which was dissolved in $\mathrm{CH}_{2} \mathrm{Cl}_{2}$. Then the organic phase was washed with $\mathrm{H}_{2} \mathrm{O}$, dried by anhydrous $\mathrm{Na}_{2} \mathrm{SO}_{4}$, and evaporated under reduced pressure. Finally, the residue was purified by silica gel-column chromatography using $\mathrm{CH}_{2} \mathrm{Cl}_{2}$-EtOAc as the eluent to give 7 as a pale yellow solid. Yield: $60 \%$, m.p. $45-46{ }^{\circ} \mathrm{C}$; ${ }^{1} \mathrm{H}-\mathrm{NMR}$ $\left(500 \mathrm{MHz}, \mathrm{CDCl}_{3}\right) \delta: 8.77(\mathrm{~s}, 1 \mathrm{H}, \mathrm{CON} \underline{\mathrm{H}}), 7.79$ (s, 2H, Ar-H), $7.46(\mathrm{~s}, 1 \mathrm{H}, \mathrm{Ar}-\mathrm{H}), 7.42$ (s, 2H, Ar-H), 6.19-6.20 (m, 1H, H-13), 5.22 (d, $J=2.2 \mathrm{~Hz}, 1 \mathrm{H}, \mathrm{H}-13)$, 4.72-4.4.77 (m, 1H, H-8), 3.11-3.13 (m, 1H, $\mathrm{H}-7), 2.48-2.51(\mathrm{~m}, 2 \mathrm{H}), 2.26-2.33(\mathrm{~m}, 3 \mathrm{H}), 2.21(\mathrm{~s}, 1 \mathrm{H}), 1.94(\mathrm{~s}, 2 \mathrm{H}), 1.51-1.73(\mathrm{~m}, 2 \mathrm{H}), 1.23(\mathrm{~s}, 1 \mathrm{H})$, 1.06 (s, 3H, H-14), 0.83-0.97 (m, 2H, H-2). MS (ESI): $m / z 389$ ([M+Na] $\left.\left.{ }^{+}\right), 100\right)$.

(3aR, 4aS, 5S, 5aR, 6aR)-5-(3-keto carbamoyl hydrazone-butyl)-5a-methyl-3-methylene-3a, 4, 4a, 5, 6, 6a-hexahydrocyclopropa[f] benzofuran-2-one (8). A mixture of 1 (223.2 mg, $0.9 \mathrm{mmol})$, semicarbazide hydrochloride $(111.5 \mathrm{mg}, 1 \mathrm{mmol})$ and sodium acetate $(176.8 \mathrm{mg}, 1.3 \mathrm{mmol})$ in distilled $\mathrm{H}_{2} \mathrm{O}(5 \mathrm{~mL})$ was stirred at $60^{\circ} \mathrm{C}$ until a white precipitate was produced, then the reaction mixture was filtered, and the filtrate was evaporated under reduced pressure. The residue was recrystallized from distilled $\mathrm{H}_{2} \mathrm{O}$ $\mathrm{MeOH}$ to give 8 as a white solid. Yield: $84 \%$, m.p. $171-173{ }^{\circ} \mathrm{C}$; IR $(\mathrm{KBr}) \mathrm{cm}^{-1}: 3317,2963,1753$, 1690, 1620; ${ }^{1} \mathrm{H}-\mathrm{NMR}\left(400 \mathrm{MHz}, \mathrm{CDCl}_{3}\right) \delta: 6.14$ (d, $\left.J=2.6 \mathrm{~Hz}, 1 \mathrm{H}, \mathrm{H}-13\right), 5.64$ (d, $J=2.4 \mathrm{~Hz}, 1 \mathrm{H}, \mathrm{H}-13), 4.88(\mathrm{~m}, 1 \mathrm{H}, \mathrm{H}-8), 4.78(\mathrm{~s}, 1 \mathrm{H},=\mathrm{NNH}), 4.60\left(\mathrm{~s}, 2 \mathrm{H}, \mathrm{O}=\mathrm{CNH}_{2}\right), 3.36(\mathrm{~m}, 2 \mathrm{H})$, $3.20(\mathrm{~m}, 1 \mathrm{H}, \mathrm{H}-7), 2.24 \sim 2.27(\mathrm{~m}, 1 \mathrm{H}), 1.97 \sim 2.13(\mathrm{~m}, 1 \mathrm{H}), 1.85(\mathrm{~s}, 3 \mathrm{H}, \mathrm{H}-15), 1.81 \sim 1.84(\mathrm{~m}, 1 \mathrm{H})$, $1.43 \sim 1.51(\mathrm{~m}, 1 \mathrm{H}), 1.04$ (s, 3H, H-14), 0.85 0.92 (m, 2H), 0.53 (m, 1H, H-5), 0.38 (m, 1H, H-1); HR-MS (ESI): $m / z$ calcd for $\mathrm{C}_{16} \mathrm{H}_{24} \mathrm{~N}_{3} \mathrm{O}_{3}\left([\mathrm{M}+\mathrm{H}]^{+}\right), 306.1812$; found, 306.1812 .

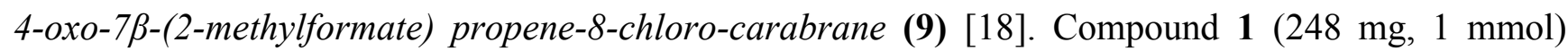
was dissolved in anhydrous $\mathrm{MeOH}(40 \mathrm{~mL})$ under reflux, and a flow of dry $\mathrm{HCl}$ was passed for $6 \mathrm{~h}$. When the starting material was nearly complete as checked by TLC, the reaction mixture was cooled to room temperature. Sodium bicarbonate $\left(\mathrm{NaHCO}_{3}, 20 \mathrm{mg}\right)$ and distilled $\mathrm{H}_{2} \mathrm{O}(30 \mathrm{~mL})$ were added to 
the above mixture, which was extracted with $\mathrm{CH}_{2} \mathrm{Cl}_{2}(50 \mathrm{~mL} \times 3)$. The organic phases were combined, and washed with $2 \%$ aq. $\mathrm{NaHCO}_{3}$, and distilled $\mathrm{H}_{2} \mathrm{O}$, dried over anhydrous $\mathrm{Na}_{2} \mathrm{SO}_{4}$, and evaporated under reduced pressure. Finally, the residue was purified by silica gel-column chromatography using petroleum ether-EtOAc as the eluent to give 9 as a colorless oily liquid. Yield: $53 \%,[\alpha]_{\mathrm{D}}{ }^{18}+49.7(\mathrm{C}$ $\left.0.35, \mathrm{CHCl}_{3}\right)$; IR $(\mathrm{KBr}) \mathrm{cm}^{-1} 2942,1756,1695,1632 ;{ }^{1} \mathrm{H}-\mathrm{NMR}\left(400 \mathrm{MHz}, \mathrm{CDCl}_{3}\right) \delta: 6.13(\mathrm{~d}$, $J=2.6 \mathrm{~Hz}, 1 \mathrm{H}, \mathrm{H}-13), 5.58(\mathrm{~d}, J=2.4 \mathrm{~Hz}, 1 \mathrm{H}, \mathrm{H}-13), 3.85$ (m, 1H, H-8), 3.76 (s, 3H, $\left.\mathrm{OCH}_{3}\right), 3.31$ (m, 2H), 2.82 (m, 1H, H-7), 2.26 2.13 (m, 2H), 1.94 (s, 3H, H-15), 1.58 1.63 (m, 2H), 1.16 (s, 3H, H-14), $0.86 \sim 0.93(\mathrm{~m}, 2 \mathrm{H}), 0.54(\mathrm{~m}, 1 \mathrm{H}, \mathrm{H}-5), 0.32$ (m, $1 \mathrm{H}, \mathrm{H}-1)$; HR-MS (ESI): $m / z$ calcd for $\mathrm{C}_{16} \mathrm{H}_{27} \mathrm{NO}_{3} \mathrm{Cl}$ $\left(\left[\mathrm{M}+\mathrm{NH}_{4}\right]^{+}\right), 316.1697$; found, 316.1695.

$(3 a R, \quad 4 a S, \quad 5 S, \quad 5 a R, \quad 6 a R)-5-(3-c h l o r o-b u t y l)-5 a-m e t h y l-3-m e t h y l e n e-3 a, \quad 4, \quad 4 a, \quad 5, \quad 6, \quad 6 a-$ hexahydrocyclopropa[f] benzofuran-2-one (10) [19]. A mixture of 2 (100 $\mathrm{mg}, 0.4 \mathrm{mmol})$ and pyridine $(0.1 \mathrm{~mL})$ in anhydrous $\mathrm{CH}_{2} \mathrm{Cl}_{2}(15 \mathrm{~mL})$ was stirred at $0{ }^{\circ} \mathrm{C}$. Thionyl chloride $\left(\mathrm{SOCl}_{2}, 0.1 \mathrm{~mL}\right)$ was added dropwise. After the addition was complete, the mixture was stirred under reflux. When the starting material was nearly completely consumed, as checked by TLC, $\mathrm{NaHCO}_{3}(20 \mathrm{mg})$ and distilled $\mathrm{H}_{2} \mathrm{O}(10 \mathrm{~mL})$ were added to the above mixture, which was extracted with $\mathrm{CH}_{2} \mathrm{Cl}_{2}(20 \mathrm{~mL} \times 3)$. The organic phases were combined, washed with $0.3 \% \mathrm{HCl}$, saturated aq. $\mathrm{Na}_{2} \mathrm{CO}_{3}$ and brine, dried over anhydrous $\mathrm{Na}_{2} \mathrm{SO}_{4}$, and evaporated under reduced pressure. Finally, the residue was purified by silica gel-column chromatography using petroleum ether-EtOAc as the eluent to produce $\mathbf{1 0}$ as colorless acicular crystals. Yield: 73\%, m.p. 97-99 ${ }^{\circ} \mathrm{C}$; IR (KBr) cm ${ }^{-1}: 2943,1757,1625,1148,628 ;{ }^{1} \mathrm{H}$ NMR $\left(400 \mathrm{MHz}, \mathrm{CDCl}_{3}\right) \delta: 6.24$ (d, $\left.J=2.6 \mathrm{~Hz}, 1 \mathrm{H}, \mathrm{H}-13\right), 5.56$ (d, $\left.J=2.4 \mathrm{~Hz}, 1 \mathrm{H}, \mathrm{H}-13\right), 4.81$ (m, 1H, H8), $4.04(\mathrm{~m}, 1 \mathrm{H}, \mathrm{H}-4), 3.14$ (m, 1H, H-7), $2.17 \sim 2.37$ (m, 2H, H-3), 1.79 1.83 (m, 2H), 1.56 (d, $J=6.0 \mathrm{~Hz}, 3 \mathrm{H}, \mathrm{H}-15), 1.43 \sim 1.46$ (m, 2H), 1.09 (s, 3H, H-14), 0.88 0.95 (m, 2H), 0.47 (m, 1H, H-5), $0.38(\mathrm{~m}, 1 \mathrm{H}, \mathrm{H}-1)$; HR-MS (ESI): $m / z$ calcd for $\mathrm{C}_{15} \mathrm{H}_{25} \mathrm{NO}_{2} \mathrm{Cl}\left(\left[\mathrm{M}+\mathrm{NH}_{4}\right]^{+}\right), 286.1568$; found, 286.1565 .

General procedure for the synthesis of compounds 11-14 [20]

A mixture of $2(100 \mathrm{mg}, 0.4 \mathrm{mmol})$ and pyridine $(0.1 \mathrm{~mL})$ in anhydrous $\mathrm{CH}_{2} \mathrm{Cl}_{2}(15 \mathrm{~mL})$ was stirred at $0{ }^{\circ} \mathrm{C}$. Acyl chloride $(0.1 \mathrm{~mL})$ in anhydrous $\mathrm{CH}_{2} \mathrm{Cl}_{2}(2 \mathrm{~mL})$ was added dropwise. After the addition, the mixture was stirred under reflux. When the reaction was nearly complete, as checked by TLC, $\mathrm{NaHCO}_{3}(20 \mathrm{mg})$ and distilled $\mathrm{H}_{2} \mathrm{O}(10 \mathrm{~mL})$ were added to the above mixture, which was extracted with $\mathrm{CH}_{2} \mathrm{Cl}_{2}(30 \mathrm{~mL} \times 3)$. The organic phases were combined, washed by $0.3 \% \mathrm{HCl}$, saturated aq. $\mathrm{Na}_{2} \mathrm{CO}_{3}$ and brine, and evaporated under the reduced pressure. Finally, the residue was purified by silica gel column chromatography using petroleum ether-acetone as the eluent to give compounds 11-14.

(3aR, 4aS, 5S, 5aR, 6aR)-5-(3-vinylCarbonate-butyl)-5a-methyl-3-methylene-3a, 4, 4a, 5, 6, 6ahexahydrocyclopropa[f] benzofuran-2-one (11). Yield: $73 \%$, a colorless oily liquid; $[\alpha]_{\mathrm{D}}^{18}+58.7(\mathrm{C}$ 0.47, $\left.\mathrm{CHCl}_{3}\right)$; IR (KBr) cm $\mathrm{cm}^{-1}: 2977,1757,1715,1642,1203 ;{ }^{1} \mathrm{H}-\mathrm{NMR}\left(400 \mathrm{MHz}, \mathrm{CDCl}_{3}\right) \delta: 6.40(\mathrm{~d}$, $\left.J=16.8 \mathrm{~Hz}, 1 \mathrm{H}, \mathrm{CH}=\underline{\mathrm{C}}_{2}\right), 6.23(\mathrm{~d}, J=2.8 \mathrm{~Hz}, 1 \mathrm{H}, \mathrm{H}-13), 6.10\left(\mathrm{~m}, 1 \mathrm{H}, \mathrm{C} \underline{\mathrm{H}}=\mathrm{CH}_{2}\right), 5.82(\mathrm{~d}$, $\left.J=10.8 \mathrm{~Hz}, 1 \mathrm{H}, \mathrm{CH}=\underline{\mathrm{C}}_{2}\right), 5.56(\mathrm{~d}, J=2.4 \mathrm{~Hz}, 1 \mathrm{H}, \mathrm{H}-13), 4.99$ (m, 1H, H-8), 4.78 (m, 1H, H-4), 3.16 (m, 1H, H-7), 2.31 2.39 (m, 2H), 1.59 1.62 (m, 2H), 1.36 1.45 (m, 2H), 1.27 (d, J=6.0 Hz, 3H, H- 
15), 1.06 (s, 3H, H-14), 0.85 0.99 (m, 2H), 0.43 (m, 1H, H-5), 0.35 (m, 1H, H-1); HR-MS (ESI): $m / z$ calcd for $\mathrm{C}_{18} \mathrm{H}_{28} \mathrm{NO}_{4}\left(\left[\mathrm{M}+\mathrm{NH}_{4}\right]^{+}\right)$, 322.2013; found, 322.2008.

(3aR, 4aS, 5S, 5aR, 6aR)-5-(3-isopropylCarbonate-butyl)-5a-methyl-3-methylene-3a, 4, 4a, 5, 6, 6ahexahydrocyclopropa[f]benzofuran-2-one (12). Yield: 72\%; a colorless oily liquid; $[\alpha]_{\mathrm{D}}^{18}+36.4(\mathrm{C}$ $\left.0.41, \mathrm{CHCl}_{3}\right)$; IR (KBr) cm ${ }^{-1}: 2974,1757,1722,1660,1148 ;{ }^{1} \mathrm{H}-\mathrm{NMR}\left(400 \mathrm{MHz}, \mathrm{CDCl}_{3}\right) \delta: 6.23(\mathrm{~d}$, $J=2.6 \mathrm{~Hz}, 1 \mathrm{H}, \mathrm{H}-13), 5.56$ (d, $J=2.4 \mathrm{~Hz}, 1 \mathrm{H}, \mathrm{H}-13), 4.95$ (m, 1H, H-8), 4.80 (m, 1H, H-4), 3.16 (m, $1 \mathrm{H}, \mathrm{H}-7), 2.51\left(\mathrm{~m}, 1 \mathrm{H}, \mathrm{C} \underline{\mathrm{H}}\left(\mathrm{CH}_{3}\right)_{2}\right), 2.30 \sim 2.38(\mathrm{~m}, 2 \mathrm{H}), 1.59 \sim 1.61(\mathrm{~m}, 2 \mathrm{H}), 1.35 \sim 1.44(\mathrm{~m}, 2 \mathrm{H}), 1.23(\mathrm{~d}$, $J=6.0 \mathrm{~Hz}, 3 \mathrm{H}, \mathrm{H}-15), 1.18\left(\mathrm{~d}, J=10.8 \mathrm{~Hz}, 3 \mathrm{H}, \mathrm{CHCH}_{3}\right), 1.14$ (d, $\left.J=11.2 \mathrm{~Hz}, 3 \mathrm{H}, \mathrm{CHCH}_{3}\right), 1.07$ (s, $3 \mathrm{H}, \mathrm{H}-14), 0.87 \sim 0.98$ (m, 2H), 0.43 (m, 1H, H-5), 0.34 (m, 1H, H-1); HR-MS (ESI): $m / z$ calcd for $\mathrm{C}_{19} \mathrm{H}_{28} \mathrm{O}_{4} \mathrm{Na}\left([\mathrm{M}+\mathrm{Na}]^{+}\right), 343.1880$; found, 343.1875 .

(3aR, 4aS, 5S, 5aR, 6aR)-5-(3-pentylCarbonate-butyl)-5a-methyl-3-methylene-3a, 4, 4a, 5, 6, 6ahexahydrocyclopropa[f] benzofuran-2-one (13). Yield: 70\%; a colorless oily liquid; $[\alpha]_{\mathrm{D}}{ }^{18}+49.4(\mathrm{C}$ $\left.0.52, \mathrm{CHCl}_{3}\right)$; IR $(\mathrm{KBr}) \mathrm{cm}^{-1}: 2935,1722,1660,1146 ;{ }^{1} \mathrm{H}-\mathrm{NMR}\left(400 \mathrm{MHz}, \mathrm{CDCl}_{3}\right) \delta: 6.23(\mathrm{~d}$, $J=2.6 \mathrm{~Hz}, 1 \mathrm{H}, \mathrm{H}-13), 5.56$ (d, $J=2.4 \mathrm{~Hz}, 1 \mathrm{H}, \mathrm{H}-13), 4.95$ (m, 1H, H-8), 4.78 (m, 1H, H-4), 3.16 (m, $1 \mathrm{H}, \mathrm{H}-7), 3.01 \sim 3.04\left(\mathrm{~m}, 1 \mathrm{H}, \mathrm{O}=\mathrm{CC}_{2} \underline{H}_{2}\right), 2.51 \sim 2.54\left(\mathrm{~m}, 1 \mathrm{H}, \mathrm{O}=\mathrm{CC}_{2}\right), 2.30 \sim 2.38(\mathrm{~m}, 2 \mathrm{H}), 1.59 \sim 1.61$ $(\mathrm{m}, 2 \mathrm{H}), 1.27 \sim 1.51(\mathrm{~m}, 8 \mathrm{H}), 1.23$ (d, $J=6.0 \mathrm{~Hz}, 3 \mathrm{H}, \mathrm{H}-15), 1.06$ (s, 3H, H-14), 0.90 0.99 (m, 2H), 0.86 0.89 (m, 3H, $\left.\mathrm{CH}_{2} \mathrm{CH}_{3}\right), 0.43(\mathrm{~m}, 1 \mathrm{H}, \mathrm{H}-5), 0.35$ (m, 1H, H-1); HR-MS (ESI): $m / z$ calcd for $\mathrm{C}_{21} \mathrm{H}_{36} \mathrm{NO}_{4}\left(\left[\mathrm{M}+\mathrm{NH}_{4}\right]^{+}\right), 366.2639$; found, 366.2632.

(3aR, 4aS, 5S, 5aR, 6aR)-5-(3-phenylCarbonate-butyl)-5a-methyl-3-methylene-3a, 4, 4a, 5, 6, 6ahexahydrocyclopropa[f] benzofuran-2-one (14). Yield: $56 \%$; a colorless oily liquid; $[\alpha]_{\mathrm{D}}{ }^{18}+73.9{ }_{(C}$ $\left.0.31, \mathrm{CHCl}_{3}\right)$; IR (KBr) cm ${ }^{-1}: 2942,1757,1711,1485,715 ;{ }^{1} \mathrm{H}-\mathrm{NMR}(400 \mathrm{MHz}, \mathrm{CDCl} 3) \delta: 8.03$ (m, 2H, H-3', 5'), 7.57 (m, 1H, H-4'), 7.46 (m, 2H, H-2', 6'), 6.24 (d, J=2.6 Hz, 1H, H-13), 5.55 (d, $J=2.4 \mathrm{~Hz}, 1 \mathrm{H}, \mathrm{H}-13), 5.18$ (m, 1H, H-8), 4.77 (m, 1H, H-4), 3.15 (m, 1H, H-7), 2.28 2.36 (m, 2H), $1.63 \sim 1.79(\mathrm{~m}, 2 \mathrm{H}), 1.42 \sim 1.56(\mathrm{~m}, 2 \mathrm{H}), 1.23$ (d, $J=6.0 \mathrm{~Hz}, 3 \mathrm{H}, \mathrm{H}-15), 1.08$ (s, 3H, H-14), 0.88 0.92 $(\mathrm{m}, 2 \mathrm{H}), 0.47$ (m, 1H, H-5), $0.35(\mathrm{~m}, 1 \mathrm{H}, \mathrm{H}-1)$; HR-MS (ESI): $m / z$ calcd for $\mathrm{C}_{22} \mathrm{H}_{30} \mathrm{NO}_{4}\left(\left[\mathrm{M}+\mathrm{NH}_{4}\right]^{+}\right)$, 372.2169 ; found, 372.2168 .

\subsection{Spore Germination Assay}

Microorganisms and maintenance: the strain of Colletotrichum lagenarium (36199) was provided by Agricultural Culture Collection of China and maintained on potato dextrose agar (PDA). Compounds 1 and 6-14 were dissolved in acetone or DMSO and added to 2\% water agar medium after sterilization to produce concentrations of $100,75,50,25,10$, and $5 \mu \mathrm{g} / \mathrm{mL}$ or $10,5,2,1,0.5$ and 0.25 $\mu \mathrm{g} / \mathrm{mL}$ of medium. Conidial suspensions $(0.2 \mathrm{~mL})$ containing $1 \times 10^{5}$ condia $/ \mathrm{mL}$, derived from cultures grown for $12 \mathrm{~d}$ on PDA plates, were spread on $2 \%$ water agar. Conidia were allowed to germinate $25 \pm 1{ }^{\circ} \mathrm{C}$ for $8 \mathrm{~h}$. Germination was quantified at three sites by counting 100 conidia per site. A conidium was scored as germinated if the germ tube had reached at least half the length of the conidium. Three plates for each concentration were used and the experiment was performed thrice, along with 98\% chlorothalonil (Syngenta Crop Protection Co., Ltd., China) as a positive control. The 
$\mathrm{EC}_{50}$ for inhibition of spore germination was calculated for each isolate. Analysis of parameters was made with the statistical analysis system (SAS institute, Inc., Cary, NC, USA) [21].

\section{Conclusions}

In summary, nine new carabrone derivatives were synthesized and evaluated in vitro against Colletotrichum lagenarium Ell et Halst. Compounds 6-8, and 12 displayed the more potent antifungal activity than 1. Meanwhile, the structure-activity relationship (SAR) demonstrated that a $\gamma$-lactone moiety was necessary for the antifungal activity of $\mathbf{1}$, and the substituents on the C-4 position of $\mathbf{1}$ could significantly affect their antifungal activity, e.g., introduction of the hydrazone substituents on the $\mathrm{C}-4$ position of $\mathbf{1}$ lead to more potent compounds.

\section{Acknowledgements}

This work was supported by a grant from the National Natural Science Foundation of China (grant No. 30971934) and the National Department Public Benefit Research Foundation of China (grant No. 200903052). We are deeply grateful for kind and helpful advice given by Tian Xuan, during the spectroscopic analysis of these compounds.

\section{References}

1. Minato, H.; Nosaka, S.; Horibe, I. Studies on sesquiterpenoids. Part VIII. The structure of carabrone, a new component of Carpesium abrotanoides Linn. J. Chem. Soc. 1964, 5503-5510.

2. Holub, M.; Samek, A.; Toman, J. Carabrone from Arnzca foliosa. Phytochemistry 1972, 11, 2627-2628.

3. Bohlmann, F.; Mahanta, P.K.; Jakupovicm, J.; Rastogi, R.C.; Natu, A.A. New sesquiterpene lactones from Inula species. Phytochemistry 1978, 17, 1165-1172.

4. Bohlmann, F.; Zdero, C.; King, R.M.; Robinson, H. Sesquiterpene lactones from Syncretocarpus sericeus. Phytochemistry 1983, 22, 1288-1290.

5. Spring, O.; Vargas, D.; Fischer, N.H. Sesquiterpene lactones and benzofurans in glandular trichomes of three Pappobolus species. Phytochemistry 1991, 30, 1861-1867.

6. Öksüz, S.; Topar, G. A eudesmanolide and other constituents fromInula graveolens. Phytochemistry 1992, 31, 195-197.

7. Mossa, J.S.; El-Feraly, F.S.; Muhammad, I.; Zaw, K.; Mbwambo, Z.H.; Pezzuto, J.M.; Fong, H.H.S. Sesquiterpene lactones and tymol esters from Vicoa pentanema. J. Nat. Prod. 1997, 60, 550-555.

8. Kim, M.R.; Lee, S.K.; Kim, C.S.; Kim, K.S.; Moon, D.C. Phytochemical constituents of Carpesium macrocephalum FR. et SAV. Arch. Pharm. Res. 2004, 27, 1029-1033.

9. Wang, F.; Yang, K.; Ren, F.C.; Liu, J.K. Sesquiterpene lactones from Carpesium abrotanoides. Fitoterapia 2009, 80, 21-24.

10. Lee, J.S.; Min, B.S.; Lee, S.; Na, M.; Kwon, B.; Lee, C.; Kim, Y.; Bae, K. Cytotoxic sesquiterpene lactones from carpesium abrotanoides. Planta Med. 2002, 68, 745-747. 
11. Maruyama, M.; Omura, S. Carpesiolin from Carpesium abrotanoides. Phytochemistry 1977, 16, 782-783.

12. Yang, C.; Shi, Y. P.; Jia, Z.J. Sesquiterpene lactone glycosides, eudesmanolides, and other constituents from Carpesium macrocephalum. Planta Med. 2002, 68, 626-630.

13. Jiang, J.W. Manuscript of Active Ingredients of Vegetable Drug; People's Health Press: Beijing, China, 1986; pp. 832-833.

14. Feng, J.T.; Zhu, M.J.; Yu, P.R.; Li, Y.P.; Han, J.H.; Shao, H.J.; Ding, H.X.; Zhang, X. Screening on the resources of botanical fungicides in Northwest China. Acta Agric. Boreali-occidentalis Sinica 2002, 30, 129-133.

15. Feng, J.T.; Zhang, Y.M.; Wang, J.R.; Zhang X. Synthesis and antifugal activities of carabrone derivatives. Chin. J. Pestic. Sci. 2007, 9, 185-188.

16. Feng, J.T.; Ma, Z.Q.; Wang, J.R.; Wang, Z.H.; Su, Z.S.; Li, G.Z.; Zhang, X. Preparation of calacane-type sesquiterpenoids separated from Carpesium macrocephalum and application as agricultural fungicide. China Patent ZL200610104867.7, 3 June, 2009.

17. Hitoshi, M.; Satoko, N.; Isao, H. Sesquiterpenoids. VIII. The structure of carabrone, a new component of carpesium abrotanoides. J. Chem. Soc., Suppl. 1964, 1, 5503-5510.

18. Li, J.; Huang, W.L.; Zhang, H.B. Synthesis of amide derivatives of andrographolide and their inhibitory activities on COX-2 expression. J. China Pharm. Univ. 2007, 38, 299-304.

19. Peng, H.; Gong, Y.F. Facile snthesis of ethyl 2-(4-hydroxyphenyl)-3,3,3-trifluoropropionate. Chin. J. Org. Chem. 2004, 24, 516-520.

20. Pamela, J.R.; Joel, R.C. Insecticidal properties of monoterpenoid derivatives to the housefly and red flour beetle. Pestic. Sci. 1994, 41, 195-202.

21. Mu, L.Y.; Wu, W.J.; Wang K.Y. Methods of plant protection with chemicals; China Agriculture Press: Beijing, China, 1994; pp. 71-78.

Sample Availability: Samples of the compounds are available from the authors.

(C) 2010 by the authors; licensee MDPI, Basel, Switzerland. This article is an Open Access article distributed under the terms and conditions of the Creative Commons Attribution license (http://creativecommons.org/licenses/by/3.0/). 